\section{Mortalidade masculina em três \\ capitais brasileiras, 1979 a 2007*}

\author{
Male mortality in three Brazilian \\ State Capitals, 1979-2007*
}

\section{Carolina Terra de Moraes Luizaga}

\section{Sabina Léa Davidson Gotlieb}

Faculdade de Saúde Pública da Universidade de São Paulo, Departamento de Epidemiologia

*Parte da dissertação de mestrado apresentada ao Programa de Pós-Graduação em Saúde Pública da Faculdade de Saúde Pública da USP (FSP/USP), intitulada "Mortalidade masculina no tempo e no espaço".

Auxílio Financeiro: Bolsista de Pós-Graduação pelo CNPq. Processo no. 134538/2008-0

Correspondência: Carolina Terra de Moraes Luizaga. Departamento de Epidemiologia, Faculdade de Saúde Pública da USP. Av. Dr. Arnaldo, 715, São Paulo, SP, Brasil, 01246-904. E-mail: carolinaterra@usp.br

\section{Resumo}

Introdução: No Brasil, verifica-se maior mortalidade masculina em praticamente todas as idades e na quase totalidade das causas. Objetivo: Estimar e descrever a tendência da mortalidade masculina, entre 1979 e 2007, em São Paulo (SP), Rio de Janeiro (RJ) e Porto Alegre (RS). Material e método: As populações de estudo referem-se aos residentes nas três capitais, nos triênios 1979/81, 1990/92, 1999/2001 e 2005/07 e respectivos óbitos. As fontes de dados incluíram Instituto Brasileiro de Geografia e Estatística e Sistemas de Informações em Saúde do Brasil. Calcularam-se os coeficientes de mortalidade gerais e específicos (brutos e padronizados). Resultados: Verificaram-se declínio da proporção de crianças e de jovens e crescimento da proporção de idosos. Até 24 anos, os homens predominaram na população; a partir daí observaram-se maiores participações femininas e razões de sexos cada vez mais baixas, evidenciando, entre idosos, maior presença de mulheres, fato associado à elevada mortalidade masculina. Houve perda intensa de jovens por causas externas. Em 2005/07, este grupo correspondeu à principal causa de morte masculina até a faixa de 40-44 anos. Nos grupos etários seguintes, as doenças circulatórias foram a principal causa. Considerações finais: As localidades evidenciam características de cidades em desenvolvimento, com redução da fecundidade, aumento da sobrevivência e envelhecimento populacional. As estimativas do elevado risco de morrer dos homens tornam clara sua vulnerabilidade, demandando ações que possibilitem redução da mortalidade por causas evitáveis, eliminando comportamentos de risco e incentivando hábitos saudáveis. Só assim haverá aumento da esperança de vida e redução das diferenças entre as mortalidades feminina e masculina.

Palavras-chave: Saúde do homem. Gênero e saúde. Mortalidade. Coeficiente de mortalidade. Sistemas de informação. Estatísticas de saúde. 


\section{Abstract}

Introduction: In Brazil, there is a higher male mortality in almost all ages and causes. The objective is to estimate and describe the trend in male mortality, between 1979/2007, in three State Capitals (São Paulo, Rio de Janeiro and Porto Alegre). Methods: The study populations refer to the residents in the three cities, in 1979/1981, 1990/1992, $1999 / 2001$ and $2005 / 2007$, and their deaths. The data source was Health Information System of the Brazilian Ministry of Health. Overall (crude/standardized) and specific mortality coefficients were calculated. Results: Up to 24 years, men predominate in the population; after, it has been observed higher female participation and gender ratios ever lower. This fact is associated with high male mortality and the intense involvement of young men with external causes. Throughout the series, these causes were responsible for large risk estimates of male death. In 2005/2007, this group was the leading cause of death in men until the age 4044 years. In the following age groups, deaths by circulatory system diseases are the main cause. Conclusion: These capitals show features of a developing city, with reduced fertility, increased longevity and consequent trend to an aging population. Estimates of the men high risk of dying make clear their vulnerability. The intensity with these events occur demand actions that will reduce the mortality rates of preventable diseases and the men's risky behaviors. It is necessary that men adopt healthier lifestyles habits, thus increasing life expectancy and reducing the gender differences in mortalities.

Keywords: Men's health. Gender and health. Mortality. Death rate. Information systems. Health statistics.

\section{Introdução}

Diferenças entre homens e mulheres vêm sendo pauta de estudos e discussões, sobretudo, no campo da saúde. São conhecidos os fatos de que os homens têm menor esperança de vida, as mulheres frequentam com maior assiduidade os serviços de saúde e autorreferem pior estado de saúde. Entretanto, elas são menos propensas a morrer, em todas as faixas etárias ${ }^{1}$. A maior sobrevida feminina está em parte associada a fatores genéticos, mas tal vantagem não equivale, obrigatoriamente, a melhor saúde. Indicadores de morbidade obtidos em inquéritos e estatísticas de demanda a serviços sugerem que as mulheres adoecem mais e apresentam prevalências mais altas de doenças crônicas não fatais ${ }^{2,3}$.

Desde 1980 tenta-se compreender o risco diferenciado de adoecer e morrer de homens e de mulheres, com vistas à promoção da saúde, mas surgem questões de direito e equidade ${ }^{4}$. Os padrões de morbimortalidade são discutidos, com interesse em determinantes biológicos, sociais e culturais associados a gênero ${ }^{2}$. A longevidade e os agravos à saúde são analisados não somente pela diferença biológica entre os sexos, mas também pelos distintos comportamentos e estilos de vida de cada um dos gêneros.

Por longo tempo os eventos masculinos foram analisados na perspectiva essencialista, como se a biologia predeterminasse o seu comportamento. Esta atitude foi superada à medida que a complexa relação entre sexo e gênero passou a ser investigada ${ }^{4}$. Para Korin ${ }^{5}$, há um modelo hegemônico de masculinidade que determina relações desiguais entre os gêneros e define o homem como ativo, competitivo, forte, capaz do trabalho físico árduo e produtivo. Ele admite ainda que, desde pequenos, eles aprendem a se desprender das qualidades ditas femininas, como passividade, enfermidade, dependência e sensibilidade. Neste modelo, o desejo e a capacidade de cuidar desaparecem na socialização do homem, obrigando-o a ter poder, autonomia, racionalidade, força e reprimir suas emoções. 
Ao cumprirem o papel masculino prescrito, comportam-se de forma inexpressiva e competitiva, o que os leva muitas vezes a apresentar condutas compensadoras, disfuncionais, agressivas e de risco.

Considera-se que os agravos à saúde estão associados às condutas específicas do homem e da mulher, nomeados fatores culturais, ocupacionais e sociais, de risco ou proteção. Não há dúvida de que existem aspectos biológicos associados às diferenças entre os sexos; um exemplo é o conjunto de complicações da aterosclerose coronariana. Após a menopausa, aumentam as mortes femininas por esta causa, diminuindo, entre os homens mais idosos, a alta preponderância desta causa de óbito em homens mais jovens ${ }^{2}$.

Desde 1992 tem havido um crescente interesse na saúde do homem na Inglaterra e no País de Gales ${ }^{6}$. Afirma-se que a saúde envolve mais do que características biológicas ou fisiológicas; no entanto, ainda não há uma definição clara e consistente do que seria saúde masculina. A organização Australian Men's Health Network define saúde masculina como "Condições ou doenças que são exclusivas dos homens, mais prevalentes e mais graves nos homens, com diferentes fatores de risco ou que necessitam intervenções distintas". O European Men's Health Forum descreve como "Questões originadas de fatores fisiológicos, psicológicos, sociais ou ambientais, com impacto sobre meninos e homens, e que requerem intervenções específicas para alcançar melhora na saúde e no bem-estar individual e populacional". Tais definições refletem a amplitude do campo e reforçam a necessidade de envolver várias disciplinas para o seu conhecimento ${ }^{7}$. A saúde masculina passou a ser discutida baseando-se nos indicadores: elevada sobremortalidade, menor expectativa de vida e menor utilização dos serviços de saúde. Além disso, os homens são mais propensos a menosprezar sinais e sintomas, protelar busca por ajuda médica e adotar comportamentos de risco, tais como tabagismo, etilismo, atitudes violentas e condução perigosa de veículos.
A descrição das mortes masculinas pode fornecer subsídios para que sejam aventadas hipóteses na explicação da mortalidade diferencial. O objetivo desse estudo é descrever a mortalidade masculina, entre 1979 e 2007, de residentes em São Paulo (SP), Rio de Janeiro (RJ) e Porto Alegre (RS), segundo faixa etária, local de residência e causa básica da morte.

\section{Metodologia}

Trata-se de estudo ecológico ${ }^{8,9}$, que analisa a série de dados no tempo e no espaço. As populações compreenderam homens residentes nas três capitais, São Paulo, Rio de Janeiro e Porto Alegre, os triênios 1979/81, 1990/92, 1999/2001 e 2005/07, e os respectivos óbitos. As variáveis selecionadas foram faixa etária, local de residência, tempo (triênios em torno do ano censitário e o último disponível, 2005/07) e causa básica de morte (9a e 10 $0^{\text {a }}$ Revisões da Classificação Internacional de Doenças ${ }^{10,11}$ ).

As fontes dos dados foram o Instituto Brasileiro de Geografia e Estatística ${ }^{12}$ e os Sistemas de Informações sobre Nascidos Vivos e sobre Mortalidade, cujos dados estão disponíveis na página eletrônica do Departamento de Informática do Sistema Único de Saúde (DATASUS) ${ }^{13}$. Coletaramse, ainda, dados socioeconômicos da base Indicadores e Dados Básicos para Saúde $(2008)^{14}$, da Rede Interagencial de Informações para Saúde.

De início construiu-se o cenário sociodemográfico das áreas para detectar possíveis diferenças. Depois foram calculados os coeficientes médios de mortalidade, gerais e específicos, para expressar as estimativas do risco de morte masculino. Foi adotada padronização direta dos coeficientes para comparações adequadas no tempo e nos locais, pois os coeficientes brutos, apesar de representarem as reais velocidade e a intensidade dos óbitos, sofrem interferência da composição etária populacional. A população padrão foi obtida pela média harmônica dos extratos etários apresentados no Censo Demográfico de 2000. 
$\mathrm{Na}$ análise da tendência, adotaram-se os triênios em torno do ano censitário para evitar distorções pontuais em virtude de possíveis erros, como subestimação do número de óbitos e/ou imprecisão de estimativas populacionais para anos afastados dos censitários, que nem sempre correspondem à realidade da área. Desta forma, os indicadores representam a mortalidade média do triênio. Os três municípios foram escolhidos por terem boa cobertura e qualidade adequada das estatísticas vitais, disponibilizando dados válidos e confiáveis ${ }^{16,17}$. A coleta foi realizada em bancos de dados não identificados, de uso e acesso públicos. Por esta razão não houve necessidade de submissão do projeto ao Comitê de Ética em Pesquisa da FSP/USP. Entretanto, os autores declararam não haver conflito de interesses.

\section{Resultados}

São Paulo, Rio de Janeiro e Porto Alegre ocupam posição de destaque no cenário nacional. São grandes centros urbanos, com adequada cobertura dos Sistemas Oficiais de Informações em Saúde do Ministério da Saúde e baixas proporções de mortes por causas mal definidas. Apresentam grande contingente populacional, taxas de urbanização, densidade demográfica e esperança de vida altas, baixa fecundidade, envelhecimento e "feminização" das populações. A taxa de crescimento vem diminuindo graças à queda da fecundidade; em 2000, chegou a valor próximo ao de reposição populacional, no Rio de Janeiro e em Porto Alegre.

Os três municípios estão em fase avançada da transição demográfica, pois seus índices de mortalidade e fecundidade alteraram-se de elevados para baixos. Este processo transformou a composição etária, tornando-a mais envelhecida (proporção de idosos acima da média nacional). As quedas da fecundidade e da mortalidade resultaram em aumento da esperança de vida. Os homens morrem em maior proporção e mais precocemente, havendo predomínio de mulheres nas idades mais avançadas.
Além de mudanças na estrutura populacional, observaram-se elevados coeficientes de mortalidade masculina. Em 1979/81, em São Paulo e Porto Alegre, os coeficientes médios brutos foram semelhantes (8 óbitos por mil homens). No Rio de Janeiro, o valor foi maior $(9,4$ por mil homens). Após a padronização, o risco de morte no Rio de Janeiro foi 6,7\% maior do que o de São Paulo e 5\% maior do que o de Porto Alegre ${ }^{13}$.

Em 1979/81, as principais causas básicas de morte nos homens foram doenças cardiovasculares (DCV) e as neoplasias. No último triênio, apesar da redução nos coeficientes padronizados (cerca de 50\%), as DCV mantiveram-se como principais. Em São Paulo e Porto Alegre, as neoplasias permaneceram na segunda posição e, na capital carioca, as causas externas se sobressaíram (Tabela 1).

As doenças do aparelho respiratório também geraram impacto na mortalidade masculina, sendo detectada, ao longo da série, maior redução em Porto Alegre. Em 1979/81, as doenças do aparelho digestivo causaram mortes em intensidade não superior a 71,2 por cem mil, e, ao final, o coeficiente não chegou a 60 por cem mil homens. Em São Paulo, no triênio inicial detectou-se o maior coeficiente padronizado por doenças infecciosas e parasitárias (56,3 por cem mil homens) e, em Porto Alegre, o menor (39,5 por cem mil homens). No triênio final, a mortalidade por doenças infecciosas dobrou em Porto Alegre, passando a 78,7 por cem mil homens e, em São Paulo, foi reduzida em $38,4 \%$ (Tabela 1 ).

Outra característica da transição epidemiológica é o declínio da mortalidade infantil. Entre 1979 a 2007, a queda mais acentuada foi em São Paulo (de 60,5 para 14 por mil nascidos vivos). No Rio de Janeiro, a redução foi de $69 \%$ (48 para 15 por mil) e, em Porto Alegre, a queda foi menor (66\%), passando de 36 para 12 por mil nascidos vivos $^{13}$. Há uma limitação a ser mencionada: em 1979/81 e 1990/92, no cálculo dos coeficientes foi usado o número de nascidos vivos registrados em cartórios, pois ainda não estava implantado o SINASC. É possível 
Tabela 1 - Coeficientes médios de mortalidade padronizados (por cem mil homens) segundo grupos de causas (capítulos da CID1), triênios e variação no tempo (\%). São Paulo, Rio de Janeiro e Porto Alegre, 1979 a 2007.

Table 1 - Average standardized mortality rates (per 100,000 males) according to underlying cause of death (chapters of ICD'), triennium and relative variation (\%), in Sao Paulo, Rio de Janeiro and Porto Alegre, Brazil, 1979 to 2007.

\begin{tabular}{lccccccccc}
\hline \multirow{2}{*}{ Causa } & \multicolumn{3}{c}{$1979 / 1981$} & \multicolumn{3}{c}{$2005 / 2007$} & \multicolumn{3}{c}{ Variação (\%) } \\
\cline { 2 - 10 } & $\mathrm{SP}^{2}$ & $\mathrm{RJ}^{3}$ & $\mathrm{POA}^{4}$ & $\mathrm{SP}^{2}$ & $\mathrm{RJ}^{3}$ & $\mathrm{POA}^{4}$ & $\mathrm{SP}^{2}$ & $\mathrm{RJ}^{3}$ & $\mathrm{POA}^{4}$ \\
\hline Infecciosas e Parasitárias & 56,3 & 50,9 & 39,5 & 38,4 & 51,8 & 78,7 & $-31,8$ & $+1,8$ & $+99,2$ \\
Neoplasias Malignas & 166,7 & 184,4 & 212,4 & 167,6 & 151,4 & 212,7 & $+0,5$ & $-17,9$ & $+0,1$ \\
Aparelho Circulatório & 506,9 & 548,5 & 497,9 & 288,9 & 260,2 & 264,0 & $-43,0$ & $-52,6$ & $-47,0$ \\
Aparelho Respiratório & 135,6 & 118,6 & 174,8 & 112,2 & 102,4 & 87,0 & $-17,3$ & $-13,7$ & $-50,2$ \\
Aparelho Digestivo & 65,7 & 64,6 & 71,2 & 59,2 & 45,1 & 48,8 & $-9,9$ & $-30,2$ & $-31,5$ \\
Causas Externas & 122,2 & 163,0 & 117,8 & 109,3 & 159,9 & 123,1 & $-10,6$ & $-1,9$ & $+4,5$ \\
\hline Número total de óbitos & 32.894 & 22.797 & 4.260 & 36.055 & 26.651 & 5.550 & $+9,6$ & $+16,9$ & $+30,3$ \\
\hline Coeficiente Geral & 1194,4 & 1150,4 & 1068,9 & 1159,5 & 1023,3 & 948,0 & $-2,9$ & $-11,0$ & $-11,3$ \\
\hline
\end{tabular}

Fonte/Source: DATASUS (dados brutos / crude data).

${ }^{1}$ Classificação Internacional de Doenças - 9a Revisão (até 1995); 10 a Revisão (a partir de 1996); ${ }^{2}$ São Paulo (SP); ${ }^{3}$ Rio de Janeiro (RJ); ${ }^{4}$ Porto Alegre (RS).

${ }^{1}$ International Classificatiom of Diseases (ICD) - 9th Revision (until 1995); 10th Revision (since 1996); ${ }^{2}$ São Paulo (SP); ${ }^{3}$ Rio de Janeiro (RJ); ${ }^{4}$ Porto Alegre (RS)

que estejam superestimados os coeficientes, devido ao fenômeno conhecido como sub-registro de nascidos vivos.

\section{Doenças Infecciosas e Parasitárias (DIP)}

Em 1979/81, entre os óbitos por DIP, a tuberculose gerou mais perdas entre residentes no Rio de Janeiro e Porto Alegre, com coeficientes padronizados de $23 \mathrm{e}$ 19 por cem mil homens. No fim da série, a mortalidade por estas causas diminuiu acentuadamente (Figura 1).
Observando a tendência de queda, esperava-se redução ainda maior na mortalidade, entre 1979 e 2007. Entretanto, com o advento da síndrome da imunodeficiência adquirida (AIDS), no início dos anos 80, o panorama foi alterado. A doença foi codificada, na CID-9a Revisão ${ }^{10}$, como deficiência da imunidade celular adquirida, fazendo parte do capítulo das Doenças das Glândulas Endócrinas, da Nutrição e do Metabolismo e Transtornos Imunitários. Em 1983, com o conhecimento da etiologia viral, os casos deveriam ter sido agrupados
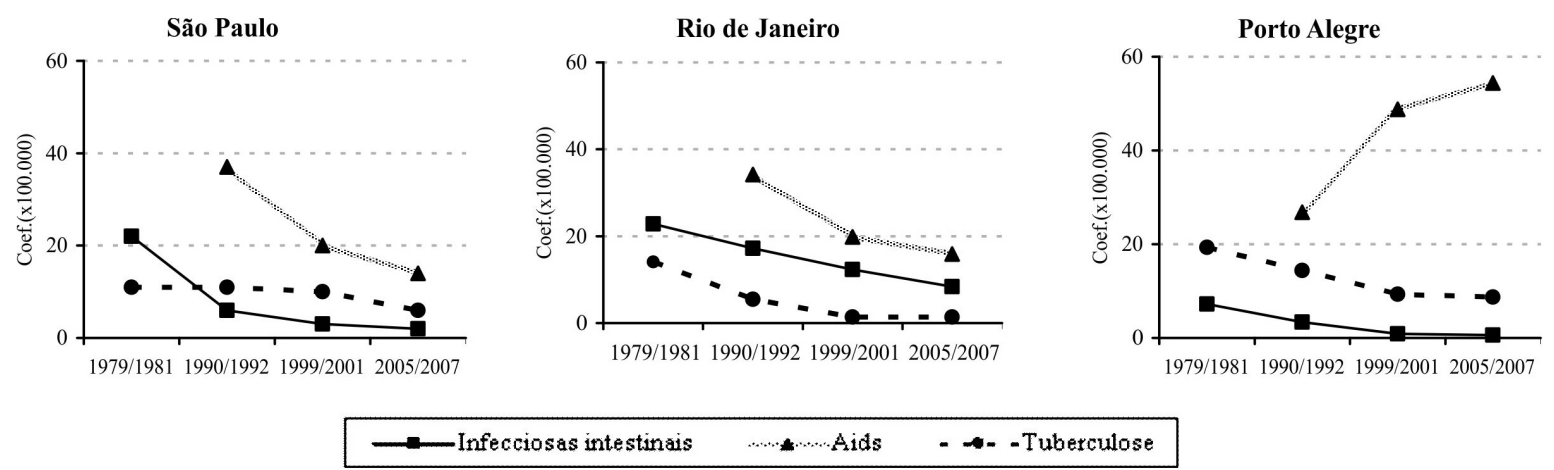

Fonte/Source: DATASUS (dados brutos/crude data).

*A AIDS, em 1990/1992, não pertencia ao capítulo das DIP.

*In 1990/1992, AIDS was not included in the IPD.

Figura 1 - Coeficientes médios de mortalidade masculina padronizados por doenças infecciosas e parasitárias segundo principais categorias* e triênio. São Paulo, Rio de Janeiro e Porto Alegre, 1979 a 2007.

Figure 1 - Average standardized mortality rates (per 100,000 males) due to infectious and parasitic diseases according to main categories * and triennium, in Sao Paulo, Rio de Janeiro and Porto Alegre, Brazil, 1979 to 2007. 
às doenças virais e classificados entre as DIP. Entretanto, pela existência de tabulações publicadas de morbidade e mortalidade, a OMS recomendou que se mantivesse a AIDS na classificação anterior, para não alterar a comparabilidade. Em 1996, com a adoção da 10a Revisão da CID $^{11}$, a AIDS tornou-se parte das DIP, resultando em aumento da mortalidade por DIP, em 1999/2001 ${ }^{18}$.

Em 1990/92, as mortes por AIDS atingiam a população masculina paulistana na intensidade de 37 óbitos por cem mil homens; no Rio de Janeiro, em 34 por cem mil e, em Porto Alegre, 27 por cem mil. No triênio seguinte, a mortalidade já havia decrescido $46 \%$ em São Paulo, $42 \%$ no Rio, e em Porto Alegre aumentou em $85 \%$. Em 2005/07, em São Paulo e no Rio de Janeiro, manteve-se a tendência de queda, sendo os coeficientes iguais a 14 e 16 por cem mil; em Porto Alegre, passou a 54 por cem mil homens (Figura 1).

\section{Neoplasias}

No início da série, a velocidade de ocorrência das mortes masculinas por neoplasias foi mais intensa em Porto Alegre (212,4 por cem mil), seguida por São Paulo, com 166,7 óbitos por cem mil homens. As principais localizações dos tumores incluíram estômago, conjunto pulmão, brônquios e traqueia, próstata e o conjunto cólon, reto e ânus. Em São Paulo, o câncer de estômago preponderou com 28 óbitos masculinos por cem mil, enquanto no Rio de Janeiro e em Porto Alegre as neoplasias de pulmão, brônquios e traqueia superaram as demais, com intensidades de 37,7 e 51 por cem mil homens. Este conjunto de tumores apareceu na segunda posição na capital paulista, sendo que, no Rio de Janeiro, a neoplasia de estômago ocupou o segundo posto, seguida pela de próstata. Em Porto Alegre, os coeficientes por cânceres de próstata e de estômago estavam na segunda e terceira posições (Figura 2).

Em 2005/07, apesar da queda de 6\% em São Paulo, o conjunto pulmão, brônquios e traqueia tornou-se a neoplasia de maior magnitude na mortalidade masculina, seguida por próstata, estômago e cólon, reto e ânus. No Rio de Janeiro e em Porto Alegre, a mesma tendência foi observada e as mortes por câncer de pulmão, brônquios e traqueia reduziram, respectivamente, em $26 \%$ e $9 \%$; apesar da discreta redução, a mortalidade em Porto Alegre manteve-se bem mais elevada. Notou-se queda na mortalidade por câncer de estômago nas três capitais, com variação entre $36 \%$ e $56 \%$. Por sua vez, as mortes masculinas pelo conjunto de cânceres de cólon, reto e ânus aumentaram em 53\%, nas capitais paulista e carioca, e em $16 \%$ em Porto Alegre (Figura 2).
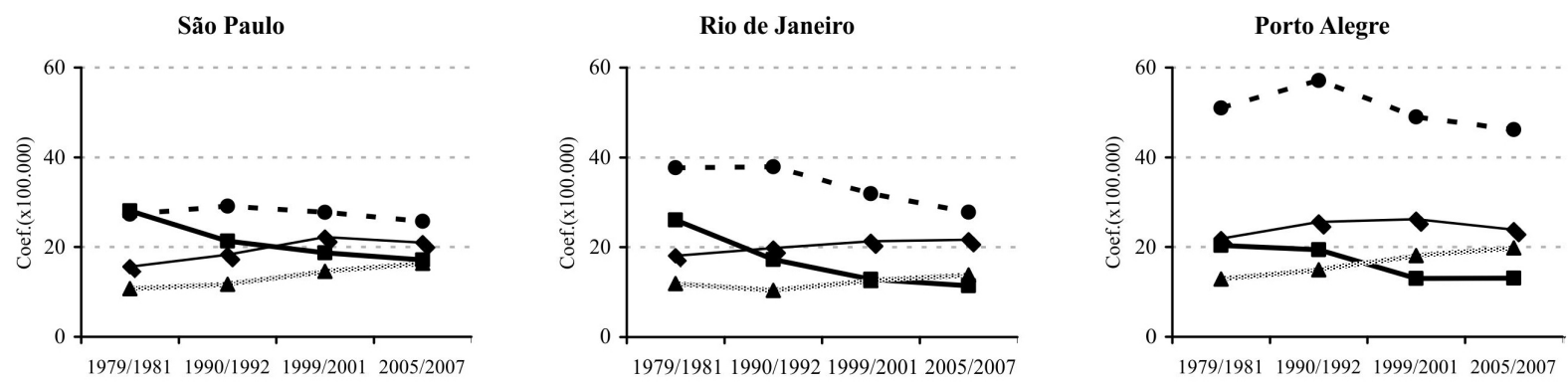

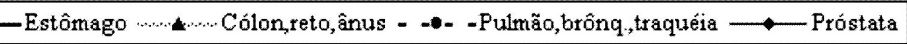

Fonte/Source: DATASUS (dados brutos/crude data).

Figura 2 - Coeficientes médios de mortalidade masculina padronizados por neoplasias segundo principais localizações anatômicas do tumor e triênio. São Paulo, Rio de Janeiro e Porto Alegre, 1979 a 2007.

Figure 2 - Average standardized male mortality rates (per 100,000 males) due to neoplasms according to main anatomical sites of the tumor and triennium, in Sao Paulo, Rio de Janeiro and Porto Alegre, Brazil, 1979 to 2007. 


\section{Doenças do Aparelho Circulatório (DAC)}

As mortes por DAC foram as mais importantes, ocorrendo em intensidade próxima a 500 óbitos por cem mil homens, em 1979/81. Até 2005/07, decresceram em quase $50 \%$ nas três capitais (Tabela 1 ). Deste grupo de causas de morte, as que mais atingiram os homens foram as doenças isquêmicas do coração (DIC), com coeficientes variando entre 207 e 222 por cem mil, em 1979/81, nos três municípios. Neste conjunto se destaca o infarto agudo do miocárdio, que apresentou tendência semelhante às DAC. Outro grupo de destaque foi o das doenças cerebrovasculares (DCV), que iniciou a série com os valores 126, 136 e 162 óbitos por cem mil homens (menor valor em Porto Alegre e o maior, no Rio de Janeiro). Seguindo a tendência de queda, as DCV reduziram cerca de $50 \%$ em São Paulo e no Rio, e 30\%, em Porto Alegre (Figura 3).

\section{Doenças do Aparelho Digestivo (DAD)}

Entre 1979 e 2007, as mortes masculinas por DAD declinaram em 10\% em São Paulo e em torno de $30 \%$ nos outros dois municípios (Tabela 1). Inicialmente, a doença alcoólica do fígado foi a maior responsável por essas mortes, com intensidades próximas a 29 por cem mil homens em São Paulo e Rio, e 36 por cem mil homens em Porto Alegre; ao longo da série, a maior redução ocorreu no Rio de Janeiro $(84,2 \%)^{13}$. Declínio foi notado, também, nas mortes por fibrose e cirrose do fígado que, no triênio inicial, geraram mortes em magnitude próxima à da doença alcoólica do fígado. No fim da série, a evolução se altera e, Porto Alegre, que apresentou o maior risco de morte, passou ao menor, após redução de $85 \%$.

\section{Causas externas}

As causas externas constituíram parcela importante das mortes masculinas. A evolução se deu de forma heterogênea entre as capitais. A maior queda, entre 1979 e 2007, ocorreu em São Paulo (11\%). No Rio de Janeiro, a redução foi de $2 \%$, Porto Alegre apresentou o menor risco, em 1979/81; porém, após aumento de $4,5 \%$, a intensidade das mortes passou a 123 por cem mil homens, sendo maior do que a estimativa dos paulistanos e menor do que a do Rio de Janeiro (Tabela 1).

Em 1979/81, o maior risco de morte masculina se deu pelos acidentes de transporte, em São Paulo e Porto Alegre (45 e 28,5 por cem mil homens). No Rio de Janeiro, neste triênio, a maior mortalidade se deu pelas agressões, com intensidade de 50 por cem mil. Em Porto Alegre, as mortes por agressões chegaram a 13 por cem mil e em São Paulo, 31 por cem mil homens.
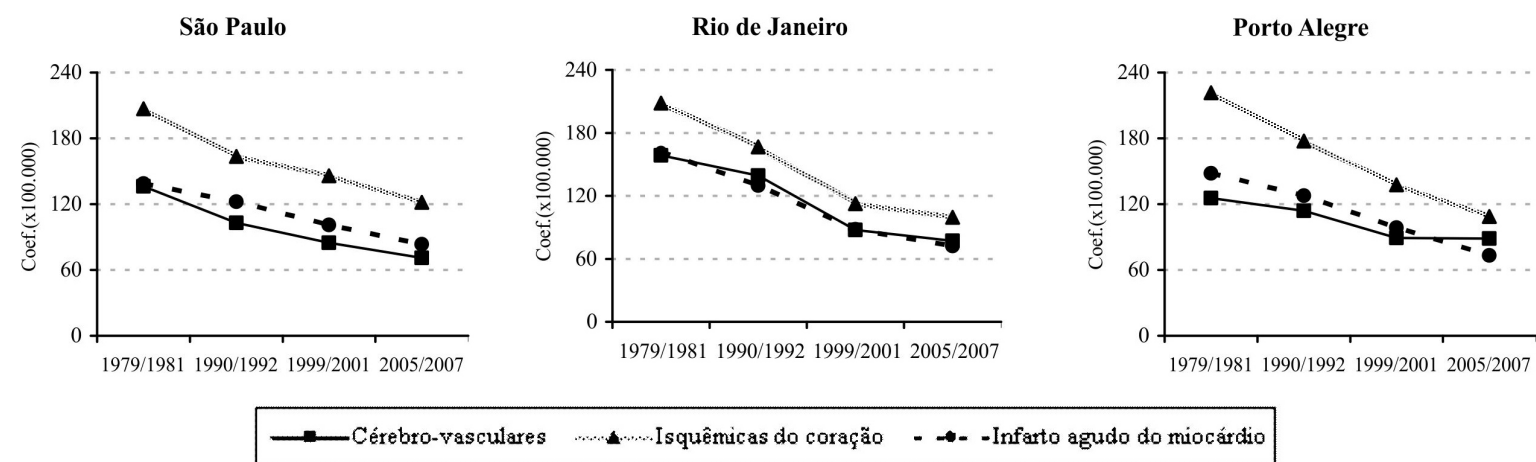

........ Isquêmicas do coração

- - Infarto agudo do miocárdio

Fonte/Source: DATASUS (dados brutos/crude data)

Figura 3 - Coeficientes médios de mortalidade masculina padronizados por doenças do aparelho circulatório segundo principais categorias e triênio. São Paulo, Rio de Janeiro e Porto Alegre, 1979 a 2007.

Figure 3 - Average standardized mortality rates (per 100,000 males) due to circulatory system diseases according to main categories and triennium, in Sao Paulo, Rio de Janeiro and Porto Alegre, Brazil, 1979 to 2007. 

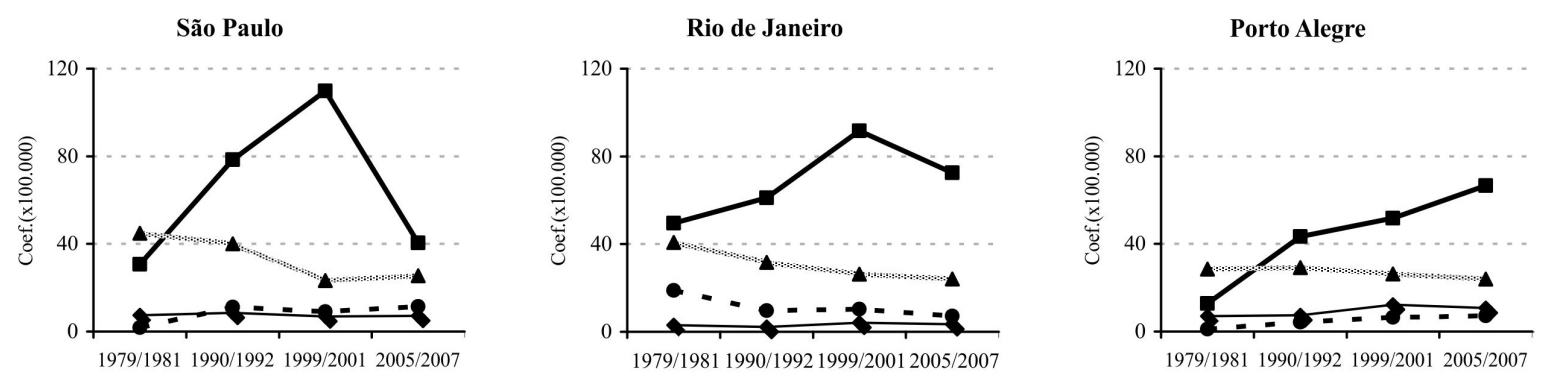

$\longrightarrow$ - Agressöbes $\ldots \ldots$ Acid de transporte - $\rightarrow$ - Quedas $\longrightarrow$ - Sucidios

Fonte/Source: DATASUS (dados brutos/crude data)

Figura 4 - Coeficientes médios de mortalidade masculina padronizados por causas externas segundo principais categorias e triênio. São Paulo, Rio de Janeiro e Porto Alegre, 1979 a 2007.

Figure 4 - Average standardized mortality rates (per 100,000 males) due to external causes according to main categories and triennium, in Sao Paulo, Rio de Janeiro and Porto Alegre, Brazil, 1979 to 2007.

Até o último triênio, houve decréscimo das mortes por acidentes de transporte, nas três capitais. A evolução das mortes por agressões mostrou expressivo aumento de $420 \%$ em Porto Alegre e de $32 \%$ e $46 \%$, respectivamente, em São Paulo e Rio de Janeiro (Figura 4).

De maneira discrepante, os suicídios geraram mais mortes masculinas em Porto Alegre, com aumento de 53\% entre 1979 e 2007. Ao fim da série, a mortalidade foi o dobro do coeficiente de São Paulo e o triplo do Rio de Janeiro. (Figura 4).

\section{Discussão}

Comparativamente ao Brasil, São Paulo, Rio de Janeiro e Porto Alegre encontram-se em fase mais avançada na transição demográfica, apresentando indicadores semelhantes aos de países desenvolvidos, isto é, mais elevadas proporções de mulheres e de idosos na população, valores maiores para as esperanças de vida ao nascer e menores taxas de fecundidade. O nível de desenvolvimento econômico e social nas três capitais é semelhante, bem como a oferta de serviços de saúde. Assim, as diferenças detectadas na mortalidade possivelmente estão relacionadas a outros fatores.

A transição demográfica gera, além de consequências sociais e econômicas, mudanças na morbidade e na mortalidade e, por conseguinte, nas condições de saúde. Devido ao rápido crescimento no número de idosos, as doenças não transmissíveis manifestam-se com maior frequência ${ }^{19}$. Este fenômeno é denominado transição epidemiológica e se caracteriza pela substituição das enfermidades tradicionais pelas não transmissíveis, resultando em expressiva redução da mortalidade infantil e elevação dos coeficientes por doenças crônicas e degenerativas ${ }^{19,20}$. As causas externas contribuem para a transformação do panorama de saúde, respondendo por grande parte de lesões e mortes, tornando-se importante problema de saúde pública.

A mortalidade padronizada masculina apresentou tendência de queda ao longo da série. As maiores estimativas de risco foram as do Rio de Janeiro, onde a queda também foi mais acentuada $(22,8 \%)$. Na capital paulista foram observados os menores valores $\mathrm{e}$ redução de $21 \%$. Em Porto Alegre, o risco de morte para homens foi reduzido em $21,5 \%$.

Entre 1979 e 2007, as três principais causas de morte masculina foram doenças cardiovasculares, neoplasias e causas externas. As cardiovasculares agiram mais intensamente a partir dos 40 anos e, entre idosos, foram notados os mais elevados coeficientes. O conjunto das neoplasias foi a segunda principal causa de morte entre meninos e idosos. As causas violentas apresentaram as estimativas mais elevadas 
de risco de morrer em adultos jovens, aparecendo como a principal causa de morte em crianças e adolescentes.

Comparando-se os coeficientes padronizados de homens e de mulheres, verificou-se que a estimativa do risco de morte masculina, no primeiro triênio, foi $57 \%, 65 \%$ e $79 \%$ maior, em São Paulo, Rio de Janeiro e Porto Alegre, respectivamente. No triênio final, as diferenças aumentaram para $74 \%, 75 \%$ e $83 \%$. O excesso de mortes masculinas tem sido explicado não só sob o enfoque biológico (maior frequência de doenças mais graves e maior fragilidade), mas também por características sociais $\mathrm{e}$ comportamentais. Biologicamente, para algumas doenças, pode-se dizer que a idade é um cofator de risco, em especial para as doenças circulatórias e neoplasias; por outro lado, fatores comportamentais como o hábito de fumar estariam contribuindo para a sobremortalidade por câncer de pulmão em homens ${ }^{2}$. Da mesma forma, a condução perigosa de veículos e o comportamento mais agressivo dos homens estariam colaborando para a maior mortalidade deles por acidentes e agressões ${ }^{1}$.

O Rio de Janeiro mostrou-se como a cidade mais violenta, com riscos ajustados de morte por causas externas mais elevados em quase todos os triênios, com exceção de 1999/2001, quando a capital paulista se sobressaiu. Na análise por idade, em 2005/07, até os 49 anos, observou-se que as mortes por agressões constituíram a principal causa externa; dos 50 aos 74 anos, houve predomínio dos acidentes de transporte e, aos 80 anos e mais, as mortes por quedas superaram as por acidentes de transporte. De maneira geral, nas três capitais, ao fim da série temporal, as mortes por agressões apresentaram-se à frente dos acidentes de transporte, sendo que em Porto Alegre o aumento verificado foi expressivo. As diferenças e semelhanças apontadas entre as localidades remetem à discussão da questão do cenário urbano como facilitador/favorecedor da violência, devido à concentração populacional e de riqueza, e à impessoalidade nas relações sociais, entre outros fatores que podem contribuir para o aumento da violência ${ }^{21}$. Estudos mais específicos são necessários para melhor entendimento da evolução dos componentes das mortes por causas externas em distintos padrões urbanos, sendo úteis na contenção do problema e na tomada de medidas preventivas.

Em relação à elevada mortalidade de adolescentes e adultos jovens por causas externas, Yunes e Zubarew ${ }^{22}$ apontaram que a morte prematura por violência gera gastos em saúde e diminui a produtividade da população. Acrescentam ainda que todas as formas de violência constituem a principal causa ao se calcularem os Anos Potenciais de Vida Perdidos. Ressaltam que a violência em jovens é evitável e prevenível, existindo alguns fatores de risco fundamentais relacionados entre si, características individuais, experiências familiares, relações interpessoais, acesso a armas, consumo de álcool e outras drogas, exposição à violência em meios de comunicação e outros fatores políticos, culturais e sociais. Segundo Laurenti e colaboradores ${ }^{2}$, a agressão tem sido apontada como a causa externa de mais difícil prevenção e controle, pois suas causas determinantes estão em geral associadas às desigualdades sociais, à existência de um estado omisso e ineficiente na adoção de políticas sociais básicas e às contradições urbanas. Como agravante, tem-se a organização do crime em torno do narcotráfico e do uso de álcool e drogas nos grandes centros urbanos. Estes fatores reforçam o conceito de que causas violentas não estão associadas a fatores biológicos, mas sim relacionadas a questões comportamentais e culturais, que contribuem para a elevada mortalidade masculina e o grande diferencial entre os sexos.

Em relação às neoplasias, ao final da série histórica o Rio de Janeiro apresentou o menor coeficiente padronizado, e Porto Alegre o maior. $\mathrm{O}$ fato de a mortalidade por câncer aumentar com o envelhecimento e, da mesma forma, o aumento da proporção de idosos na população e a redução da mortalidade por doenças tradicionais, têm levado alguns autores a discutir se o aumento 
observado é real ou aparente. De acordo com San Martin, citado por Laurenti e colaboradores ${ }^{2}$, a dúvida consiste na questão se o aumento é devido ao envelhecimento da população ou é consequência de melhores técnicas diagnósticas, ou até à maior procura por tratamentos mais eficientes e melhor certificação das causas de morte.

Cabe destacar que a mortalidade masculina por neoplasias também excedeu a feminina (em pouco mais de $50 \%$ ) ao longo da série e nas três capitais. Analisando-se alguns sítios de tumores mais importantes no homem, verificaram-se altas estimativas de risco para pulmão, brônquios e traqueia, próstata e estômago. Os mesmos achados foram comentados por Hallal et al. ${ }^{23}$, no Rio Grande do Sul, e em publicação do Ministério da Saúde ${ }^{24}$ para o Brasil. A mortalidade por tumores de pulmão, brônquios e traqueia apresentou pequenas variações entre 1979 e 2007. Já é conhecida a sua associação com o tabagismo e a maior incidência de casos e óbitos entre homens. Em 2005/07, na capital paulista, os coeficientes médios de mortalidade variaram de 5 por cem mil homens de 40 a 44 anos até 311 por cem mil homens de 80 anos e mais; no Rio de Janeiro, nessas idades foram de 6 a 273 por cem mil e, em Porto Alegre, de 7 a 507 por cem mil homens. A maior intensidade de mortes masculinas em Porto Alegre condiz com a conhecida maior prevalência masculina de tabagismo lá existente ${ }^{14}$. Esta expressiva mortalidade foi também observada em publicação da RIPSA ${ }^{14}$ e para a população masculina do Rio Grande do Sul, no estudo de Malta et. al. (2007) ${ }^{25}$.

A neoplasia da próstata passou a ter importância maior a partir dos 40 anos; entretanto, é entre os idosos que se encontram os coeficientes mais elevados. As campanhas de prevenção têm sido intensificadas em face da maior universalização dos exames preventivos e das elevadas estimativas de risco masculino, em tendência crescente. A mortalidade por câncer gástrico declinou ao longo da série, nas três capitais; esta tendência já era apontada por Fonseca ${ }^{26} \mathrm{e}$ Latorre $^{27}$. A etiologia deste tipo de câncer não é bem conhecida, mas Fonseca ${ }^{26}$ cita a troca de algumas práticas tradicionais de conservação dos alimentos (uso de sal e defumação) pela refrigeração, que seria fator protetor. Hábitos como a ingestão de alimentos gordurosos e industrializados e a bactéria Helicobacter pylori estão mencionados entre os fatores de risco ${ }^{28}$.

Chamou atenção a mortalidade por câncer de esôfago em Porto Alegre, que, na sequência de triênios, apresentou coeficientes médios padronizados de 15, 13, 12 e 11 por cem mil. Comparando estes valores com os verificados nas outras capitais, há discrepâncias importantes, pois, na capital paulista, no início, a mortalidade foi de 11 óbitos por cem mil, mas nos triênios seguintes não passou de 9 por cem mil. No Rio de Janeiro, inicialmente de foi 8 por cem mil e, depois, 6 por cem mil homens. Há estudos relacionando a incidência de câncer de esôfago com o consumo de erva-mate ${ }^{29}$. A infusão quente, feita com folhas secas e picadas de Illex paraguayensis, tem sido considerada como possível causa de câncer esofágico na América do Sul, pois elevadas incidências são observadas em áreas que incluem o Sul do Brasil, Uruguai e Nordeste da Argentina. Há dois possíveis mecanismos para o mate elevar o risco de carcinoma de esôfago; um é o extrato da planta Ilex paraguayensis, que teria substâncias carcinogênicas; mas, até agora, não houve aceitação desta hipótese. Outra suspeita seria a de o mate não ter substâncias carcinogênicas, mas a elevada temperatura em que é consumido potencializaria a carcinogênese, em associação com o consumo de álcool e tabaco ${ }^{29}$.

Em relação às doenças não transmissíveis, é de se destacar que, apesar da tendência decrescente, as mortes pelas DAC ocuparam a primeira posição dentre as principais causas de morte nos três municípios e no conjunto de triênios, em todos os grupos etários a partir dos 45 anos. As DAC, as neoplasias malignas e as causas externas constituíram as principais causas de morte masculina, oscilando a posição em função da faixa etária, localidade e triênio. Estes achados foram semelhantes aos descritos 
em outros estudos ${ }^{24,30-32}$. Sabe-se que as doenças não transmissíveis são de etiologia multifatorial e compartilham fatores de risco em comum (tabagismo, inatividade física, dieta inadequada, obesidade, etilismo, entre outros), de natureza comportamental, que podem ser modificados ${ }^{33,34}$. Estas doenças demandam assistência continuada de serviços e custos progressivos, tendo em vista o envelhecimento da população e a mudança nos padrões de morbidade e mortalidade ${ }^{35}$.

Embora tenha havido grande participação das doenças crônicas, degenerativas e por violência na mortalidade de homens, foi possível observar que as DIP, em 1979/81, constituíram importante causa de mortalidade, mas em menor intensidade do que representavam décadas atrás. Observou-se redução da mortalidade por DIP entre 1979 e 1992 nas três capitais. No entanto, em 1999/2001, os coeficientes voltaram a crescer até que, em 2005/07, à exceção de Porto Alegre, a mortalidade por DIP foi menor que a do triênio anterior, mas, ainda assim, em intensidade maior que a de 1990/92. Se não fosse a inclusão da AIDS entre as DIP, em 1996, sua queda poderia ter sido mais acentuada.

AAIDS, na década de 80, esteve associada ao homem, especialmente aos homossexuais. Apesar do crescente número de casos por transmissão heterossexual, a incidência da doença continuava sendo maior em homens $^{2}$, o que pode ser percebido quando se analisa a sobremortalidade masculina no tempo. Em 1990/92, a mortalidade por AIDS em homens foi $517 \%$, $580 \%$ e $671 \%$ maior que a das mulheres, em São Paulo, Rio de Janeiro e Porto Alegre. Ao final da série temporal, os percentuais do excesso de mortes masculinas estavam reduzidos a $180 \%$, $200 \%$ e $170 \%$. Fato marcante é o aumento na mortalidade por AIDS, em Porto Alegre, que em 2005/07, na faixa de 15 a 19 anos, foi 3 e 4 vezes maior que a verificada nas outras capitais. Nas demais idades, na comparação entre Porto Alegre e São Paulo, as razões foram entre 3 e 7 , chegando a 11 vezes no grupo de 75 a 79 anos. Tanto o Rio Grande do
Sul, como sua capital vêm apresentando os mais elevados coeficientes de mortalidade pela doença no país ${ }^{14}$. Em 2005, segundo a RIPSA $^{14}$, a região Sul apresentou mortalidade masculina por AIDS $17 \%$ maior que a do Sudeste. Ainda não foram identificadas as causas da maior mortalidade por AIDS na região Sul do Brasil. A grande variabilidade genética dos subtipos circulantes do vírus da imunodeficiência humana (HIV) e a alta prevalência de soropositivos infectados pelo subtipo $\mathrm{C}$ nesta região diferem do que ocorre na maior parte do país. Desta forma, há em andamento estudos que avaliam a resposta ao tratamento com antiretrovirais, principalmente nesta região, pois diferentes subtipos virais podem ter respostas distintas aos diversos tratamentos ${ }^{36,37}$.

Os achados obtidos evidenciam um excesso de mortes masculinas por causas evitáveis. Parte dessas causas mostrou que, além do fator biológico, há associação com o comportamento masculino e o seu estilo de vida. Investigações mais detalhadas são necessárias, mas o panorama descrito da mortalidade em homens serve de base para o desenvolvimento de políticas e ações específicas para a promoção da saúde masculina, no Brasil.

\section{Considerações finais}

Além da cuidadosa escolha dos dados, considerou-se necessária a padronização dos coeficientes de mortalidade das três áreas para que pudessem ser comparados. Este procedimento nem sempre é utilizado, restringindo o número de estudos comparáveis no tempo e entre diferentes localidades.

Considerando que a maior parte dos óbitos masculinos tem como causa básica doenças crônicas, degenerativas e violências, e que a sua ocorrência é em parte atribuída a fatores comportamentais e culturais, cabe ressaltar que investimentos em prevenção são fundamentais e deverão surtir efeitos positivos. Estudos epidemiológicos são úteis na identificação dos fatores e entendimento dos mecanismos que levam os homens a apresentarem coeficientes 
mais elevados de mortalidade. Algumas hipóteses aventadas explicariam boa parte dessas diferenças. Além de fatores genéticos e hormonais, sabe-se que alguns determinantes biológicos, sociais e comportamentais ligados ao gênero implicam em riscos diferenciados de adoecer e morrer entre homens e mulheres.

O modelo hegemônico de masculinidade encoraja homens a adotarem comportamento mais agressivo e de risco, tais como direção perigosa de veículos, elevadas prevalências de tabagismo, consumo de álcool e outras drogas, dieta rica em gorduras e menor preocupação com a saúde. Por outro lado, há traços culturais desencorajando-os a apresentar atitudes de passividade, sensibilidade e fragilidade, por estas serem consideradas tipicamente femininas. Decorre, então, que os homens utilizam muito menos os serviços de saúde e acabam desprezando os primeiros sinais e sintomas das doenças. Tais atitudes os predispõem à maior ocorrência de violência, lesões, doenças e morte precoce.

Paralelamente às ações de prevenção, deve haver motivação para que os homens passem a fazer maior uso dos serviços de saúde levando à viabilização de diagnóstico precoce e ampliação do acesso ao tratamento, traduzindo-se na redução das mortes e do perverso diferencial de saúde entre sexos.

\section{Referências}

1. Case A, Paxson C. Sex differences in morbidity and mortality. Demography 2005; 42(2):189-214.

2. Laurenti R, Buchalla CM, Mello Jorge MHP, Lebrão ML, Gotlieb SLD. Perfil epidemiológico da saúde masculina na Região das Américas: uma contribuição para o enfoque de gênero. São Paulo: Centro Colaborador da OMS para a Classificação de Doenças em Português; 1998.

3. Gómez EG. Equidad, Género y Salud [documento publicado na Internet] marzo 2001. División de Salud y Desarrollo Humano, Programa Mujer, Salud y Desarrollo. Organización Pan-Americana de la Salud/ Organización Mundial da Saúde. Disponível em: http://www.eclac.cl/mujer/noticias/noticias/4/8274/ equigensa1.PDF. [Acessado em 20 de setembro de 2008].

4. Schraiber LB, Gomes R, Couto MT. Homens e saúde, na pauta da Saúde Coletiva. Ciênc Saúde Coletiva 2005; 10(1): 7-17.

5. Korin D. Nuevas perspectivas de género en salud. Adolesc Latinoam 2001; 2(2): 67-9.

6. Luck M, Banford M, Williamson P. Men's Health: perspectives, diversity and paradox. Reino Unido: Blackwell Publishing; 2000. Disponível em: http:/ /books. google.com/books?printsec=copyright\&lr=\&id=Q1y8UJi ZBQUC\&hl=pt-BR\#PPP1,M1 [Acessado em 12 janeiro de 2009]

7. White A. Men's health: the challenges ahead. The Journal of Men's Health \& Gender [periódico na internet] (periódico renomeado para Journal of Men's Health) 2004; 1(4): 296-9. Disponível em: http:// www.sciencedirect.com/science?_ob=ArticleURL\&_ udi=B7GW7-4DXKH93-7\&_user $=5674931 \& \_r d o c=1 \&$ $\mathrm{fmt}=$ \&_orig=search\&_sort $=\mathrm{d} \& \mathrm{view}=\mathrm{c} \& \_$acct $=$
C000049650\&_version $=1$ \&_urlVersion $=0$ \&_userid $=56$ 74931\&md5=8da711b6f7e61607a3ba95e132d74ab2. [Acessado em 14 dezembro de 2008]

8. Morgenstern H. Ecologic studies in Epidemiology: Concepts, Principles and Methods. Ann Rev Public Health 1995, 16: 61-81.

9. Gordis L. Epidemiologia. Rio de Janeiro; Revinter; 2004.

10. OMS. Classificação Internacional de Doenças. 9a Revisão. São Paulo: Centro da OMS para Classificação de Doenças em Português. Ministério da Saúde/USP; 1975.

11. Organização Mundial da Saúde. Classificação Estatística Internacional de Doenças e Problemas Relacionados à Saúde, 10 Revisão. São Paulo: Centro Colaborador da Organização Mundial da Saúde para Classificação de Doenças em Português: USP, 1995.

12. Instituto Brasileiro de Geografia e Estatística [base de dados na Internet]. Censos demográficos do Brasil, 1980, 1991 e 2000. Disponível em: http:/ /www.ibge.gov.br [Acessado em 10 novembro de 2008]

13. Departamento de Informática do SUS [base de dados na Internet]. Brasília (DF): Ministério da Saúde. Disponível em: http:/ / www.datasus.gov.br [Acessado em 15 janeiro de 2009]

14. Rede Interagencial de Informações para a Saúde. Indicadores e Dados Básicos 2008 [base de dados na Internet]. Brasília: Organização Pan-Americana da Saúde. Disponível em: http://tabnet.datasus.gov.br/cgi/ idb2008/matriz.htm?saude=http\%3A\%2F\%2Ftabnet. datasus.gov.br\%2Fcgi\%2Fidb2008\%2Fmatriz.htm\&bo taook=OK\&obj=http\%3A\%2F\%2Ftabnet.datasus.gov. br\%2Fcgi\%2Fidb2008\%2Fmatriz.htm. [Acessado em 18 janeiro de 2009]. 
15. Laurenti R, Mello Jorge MHP, Lebrão ML, Gotlieb SLD. Estatísticas de Saúde. 2 ed. rev. e atual. São Paulo: EPU; 2005.

16. RIPSA. Rede Interagencial de Informações para a Saúde. Indicadores e Dados Básicos (IDB) /Ripsa. Brasília: Organização Pan-Americana da Saúde; 2004.

17. Laurenti R, Mello Jorge MHP, Gotlieb SLD. O Sistema de Informações sobre Mortalidade: passado, presente e futuro. São Paulo: Centro Colaborador da OMS para a Família de Classificações em Português; 2006. 107 p. (Série divulgação, n.11).

18. Buchalla CM, Barbieri DD, Laurenti R, Teixeira PR, Reis RAO, Lima LOO. A AIDS/SIDA: as estatísticas de mortalidade como fonte de informações. São Paulo: Centro da OMS para Classificação de Doenças em Português; 1990. (Série Divulgação, n. 6).

19. Jamison D. Disease control priorities in developing countries. New York: Oxford University Press; 2006.

20. Bobadilha JL, Frenk J, Lozano R, Frejka T \& Stern C. The epidemiological transition and health priorities In: $D T$ Janison Disease control priorities in developing countries. Oxford Medical Publications: Oxford University Press, Oxford; 1993. pp. 161-87.

21. Mello-Jorge MHP, Gawryszewski VM, Latorre MRDO. Análise dos dados de mortalidade. Rev. Saúde Pública, 31 (4 Suplemento): 5-25, 1997.

22. Yunes J, Zubarew T. Mortalidad por causas violentas en adolescentes y jóvenes: un desafio para la Región de las Américas. Rev. bras. Epidemiol. Vol.2, n.3, pp. 102-171, 1999.

23. Hallal ALC, Gotlieb SLD, Latorre MRDO. Evolução da mortalidade por neoplasias malignas no Rio Grande do Sul, 1979-1995. Rev Bras Epidemiol 2001; 4(3): 168-77.

24. Saúde Brasil 2007 - Uma análise da situação de saúde. Secretaria de Vigilância em Saúde. Departamento de Análise de Situação de Saúde. Brasília: Ministério da Saúde; 2008.

25. Malta, DC et al. Tendência de mortalidade do câncer de pulmão, traqueia e brônquios no Brasil, 1980-2003. J Bras Pneumol 2007; 33(5): 536-43.

26. Fonseca LAM. Mortalidade por câncer no Estado de São Paulo: perfil atual, distribuição geográfica e tendências temporais (tese de doutorado). São Paulo: Faculdade de Saúde Pública da USP; 1996.

27. Latorre MRDO. A mortalidade por câncer de estômago no Brasil: análise do período de 1977 a 1989. Cad Saúde Pública 1997; 13(S1): 67-78.

28. Forman D, Newell DG, Fullerton F, Yarnell J W, Stacey R, Wald N, Sitas F. Association between infection with Helicobacter pylori and risk of gastric cancer: evidence from a prospective investigation. BMJ 1991; 302: 1302-5.
29. Barros SGS, Ghisolfi ES, Luz LP, Barlem GG, Vidal RM, Wolff FH et al . Mate (chimarrão) é consumido em alta temperatura por população sob risco para o carcinoma epidermóide de esôfago. Arq Gastroenterol 2000; 37(1): 25-30.

30. White A e Cash K. A Report on the State of Men's health across 17 european countries. School of Health \& Community Care, Leeds Metropolitan University. England: The European Men's Health Fórum, 2003. Disponível em: http://www.emhf.org/resource_images/ EMHF_Report_part_1.pdf. [Acessado em 20 de fevereiro de 2009]

31. Laurenti R, Mello Jorge MHP, Gotlieb SLD. Perfil epidemiológico da morbimortalidade masculina. Ciência e Saúde Coletiva 2005; 10(1): 35-46.

32. White A, Holmes M. Patterns of mortality across 44 countries among men and women aged $15-44$ years. $J$ Mens Health Gend 2006; 3(2): 139-51.

33. Willet WC, Koplan JP, Nugent R, Dusenbury C, Puska P, Gaziano TA. Prevention of chronic disease by means of diet and lifestyle changes. In: Jamison DT, Breman JG, Measham, Measham AR, Alleyne G, Evans DB, Jha P, Mills A et al. Disease control priorities in developing countries. World Bank; 2006. pp 833-50.

34. Malta DC, Cezário AC, Moura L, Morais Neto OL, Silva Jr JB. A construção da vigilância e prevenção das doenças crônicas não transmissíveis no contexto do Sistema Único de Saúde. Epidemiol Serv Saúde 2006; 15(1): 4765.

35. Achutti A, Azambuja MIR. Doenças crônicas nãotransmissíveis no Brasil: repercussões do modelo de atenção à saúde sobre a seguridade social. Ciênc Saúde Coletiva 2004; 9(4): 833-40.

36. Inocêncio LA, Pereira AA, Sucupira MC, Fernandez JCC, Jorge CP, Souza DFC et al. Brazilian Network for HIV Drug Resistance Surveillance: a survey of individuals recently diagnosed with HIV. J Int AIDS Soc 2009; 12: 20.

37. Ministério da Saúde, s.d. Departamento de DST/AIDS e hepatites virais. Projeto de pesquisa: Caracterização do polimorfismo do HIV-1 de subtipos B e C e seu impacto na susceptibilidade aos inibidores de protease em pacientes em tratamento na região Sul do Brasil. Coordenador: Tomoko Sasazawa Ito. Disponível em: http://sistemas.aids.gov.br/ct/projetos/redes_ pesquisa4.asp?ano=2007\&numero=279. [Acessado em 20 de março de 2010]

Recebido em: 03/02/11 Versão final apresentada em: 14/04/11 Aprovado em: 11/05/11 